\title{
DESAFIOS METODOLÓGICOS DA INCORPORAÇÃO DE FERRAMENTAS COMPUTACIONAIS PARA ANÁLISES QUALITATIVAS
}

\author{
Glaucia Santos Marcondes*
}

\begin{abstract}
Resumo: Os avanços na tecnologia de informática têm alterado as condições de produção e divulgação do conhecimento científico. Dentro das Ciências Sociais, redes de sociabilidade virtuais, blogs e outras formas de comunicação via internet se unem às iconografias, vídeos, áudios, diários, registros manuscritos entre outros documentos e materiais comumente analisados pelos pesquisadores. Tanto a incorporação dessas novas formas documentais quanto de ferramentas como programas de computador que auxiliam no armazenamento, organização e análise desses materiais tem desafiado os pesquisadores a alterar não apenas a maneira de trabalho, mas igualmente a desenvolver renovados olhares críticos sobre técnicas e métodos de análise, independente da adoção de uma perspectiva quantitativa ou qualitativa. Como qualquer inovação científica, seja ou não tecnológica, desperta desconfianças, resistências e empolgações, renovando com novas e velhas inquietações não somente a discussão mais restrita sobre métodos e técnicas, como também epistemológica. Esse artigo buscará particularmente refletir sobre a utilização de softwares desenvolvidos para a análise de dados qualitativos, visando destacar os principais pontos de resistências e inovações que esse tipo de tecnologia tem suscitado no debate sobre metodologias de pesquisa nas áreas das Ciências Sociais.
\end{abstract}

Palavras-chave: Metodologia, pesquisa qualitativa, CAQDAS.

Abstract: Advances in computer technology have changed the production and dissemination of scientific knowledge. Increasingly, social networks sites, blogs and other forms of internet communication become materials for qualitative research in the Social Sciences as well as the traditional iconography, video, audio, diaries,

\footnotetext{
* Doutora em Demografia. Bolsista do Programa Nacional de Pós-Doutorado da Capes (PNPD/CAPES) junto ao Programa de Pós-Graduação em Demografia do IFCH/Unicamp.
} 
$|72|$

Desafios metológicos da incorporação...

handwritten records and other documents. The incorporation of these new document types and computer programs that assist in storing, organizing and analyzing these sources has forced researchers to change the way they work and the knowledge about techniques and analysis methods, to conduct quantitative or qualitative research. Like any scientific innovation, whether technological or not, arouses suspicion, resistance and expectations, renewing with new and old concerns not only the narrower discussion on methods and techniques, as well as epistemologically. This article aims to reflect on the use of computer software for qualitative data analysis (CAQDAS), aiming to highlight the points of resistance and innovations that this type of technology has raised in the discussion of research methodologies in the areas of Social Sciences.

Keywords: Methodology, qualitative research, CAQDAS.

As mudanças provocadas pela ampla disseminação de tecnologias computacionais na produção e divulgação do conhecimento científico são inegáveis. No entanto, ainda está muito longe de se esgotar a necessidade de reflexões críticas a respeito dos impactos que essas tecnologias têm e ainda terão sobre os modos de se pensar e fazer ciência nos tempos atuais (Santos 2001; Baumgarten, Teixeira, Lima, 2007; Dwyer, 2005; Silva, 1998). Para abordar essa questão, esse artigo reflete particularmente sobre o uso de programas de computadores que visam auxiliar na produção de análises qualitativas nas áreas das Ciências Sociais. Mesmo que de forma breve, destacar-se-á os principais pontos do debate metodológico acerca da incorporação desse tipo de ferramental nas pesquisas de cunho qualitativo, associadas a um modus operandis que privilegia o envolvimento direto, a imersão e o olhar contextualizado e integral do pesquisador com relação as suas fontes de pesquisa.

Os debates metodológicos são imprescindíveis para o desenvolvimento do conhecimento científico por colocarem em evidência as formas pelas quais o entendimento sobre o real é construído, identificando que tipo e de que maneiras eventos ou fenômenos dessa realidade têm sido sistematicamente observados, descritos e analisados. Não apenas isso, mas também, fazendo questionamentos sobre o papel que o pesquisador exerce na 
construção desse conhecimento. Essa discussão adquire particular complexidade quando o objeto do conhecimento é a própria sociedade.

Dado o caráter multidimensional, relacional, de grande complexidade e imprevisibilidade da vida social, os pressupostos que informam o paradigma científico positivista - objetividade, regularidade, linearidade, redução, tendência à estabilidade - que fundamenta o desenvolvimento da ciência moderna, calcada nas ciências naturais, implicaria um tipo de apreensão reducionista, sincrônica e inadequada da vida social. Se o mundo social é entendido como resultado de uma construção com significados e símbolos, ou seja, é um artefato humano, sua compreensão demanda justamente pelo exame dessa construção e dos seus significados. (Baumgarten, Teixeira, Lima, 2007) Dessa maneira, as preocupações que mobilizam os cientistas sociais não dizem respeito à busca por formulações de leis generalizantes, mas dos significados das ações sociais (Goldenberg, 2004). Essa ideia fundamenta o paradigma compreensivo, que dá forma às metodologias qualitativas, associadas a arcabouços teóricos que procuram lidar com as subjetividades, os significados, símbolos e linguagens construídos e partilhados socialmente (Goldenberg, 2004; Marcondes, 2010). Informados por esses paradigmas, estratégias de quantificação ou de qualificação do material empírico são colocadas comumente não apenas como diferentes, mas como opostas, e até mesmo excludentes, gerando discussões do tipo: métodos qualitativos versus métodos quantitativos (Marcondes, 2010; Fazito, 2009).

Contemporaneamente, as questões que cercam esse debate se renovam com a incorporação das chamadas "metodologias informacionais" (Baumgarten, Teixeira, Lima, 2007; Santos, 2001; Teixeira e Becker, 2001; Dwyer, 2004) que dizem respeito aos usos e impactos das tecnologias de informação e comunicação na produção do conhecimento científico ${ }^{1}$. Particularmente na

1 Baltar e Baltar (2010) consideram equivocada a utilização do termo "metodologias informacionais" por implicitamente levar a suposição de 
$|74|$

Desafios metológicos da incorporação...

Sociologia, a discussão procura avançar na reflexão das relações entre produção de conhecimento e sociedade da informação (Baumgarten, Teixeira, Lima, 2007; Santos, 2001; Teixeira e Becker, 2001).

Assim, ao se observar esse conjunto de questões sobre teorias, métodos e técnicas no interior das Ciências Sociais, com frequência a utilização de programas de computador para análise de dados está associada a pesquisas de cunho quantitativo (Guizzo, Krziminski, Oliveira, 2003). Os pacotes estatísticos (SPSS, STATA, SAS) que permitem o manuseio e tratamento analítico de bancos de dados numéricos e categóricos são bem conhecidos². E não raro ainda fazem pesquisadores iniciantes e iniciados perderem noites de sono até se familiarizarem com os recursos oferecidos por esses. Quando o assunto se volta para pesquisas com abordagens qualitativas, que se caracterizam por dados não numéricos, discursivos e estruturados de forma não rígida, os programas de computador existentes para tratamento analítico desse tipo de material não são tão populares, mesmo diante do crescente número de usuários e maior sofisticação de recursos alcançados por esses programas ao longo da última década. (Lage, 2011). Basta um breve exame dos manuais de metodologia de pesquisa qualitativa para obtermos orientações em detalhe sobre planejamento, execução e interações em campo; técnicas de abordagem, entrevista e observação; tipos de dados e métodos de

que os usos de técnicas de informática constituiriam um novo "campo do saber", quando na verdade o que existem são apenas programas, recursos ou ferramentas de informática que se inserem nas praticas de ensino e pesquisa.

2 Vale destacar também o interesse crescente por Programas de Georeferenciamento - ArcGIS, Spring, AutoCAD, MapInfo, Terra View, entre outros... - que se aliam ao processo de análise de dados quantitativos, possibilitando explorar formas alternativas de visualização e aprofundamento dos resultados de pesquisa. 
análises. Raramente se encontra informações detalhadas sobre o uso de pacotes computacionais ${ }^{3}$.

Muito embora os chamados Computer Aided Qualitative Data Analysis Software (CAQDAS) ${ }^{4}$ tenham começado a circular entre cientistas sociais britânicos e americanos nos anos de 1980, no Brasil os primeiros relatos de pesquisa utilizando esse tipo de ferramenta surgem apenas na segunda metade da década de 1990. Da divulgação e incorporação desses programas no campo das Ciências Sociais, destacadamente na Sociologia, emergem tanto desconfianças ${ }^{5}$ quanto empolgadas expectativas (Teixeira e Becker, 2001; Moreira, 2007; Lage, 2011), que têm alimentado novas e velhas inquietações não somente no que se refere aos métodos e

\footnotetext{
${ }^{3} \mathrm{Na}$ maioria dos casos os manuais estão em língua inglesa, ver alguns exemplos: BAUER, Martin W.; GASKEL, Georg (eds.). Qualitative Researching with text, image and sound. London: Sage Publications, 2000. SILVERMAN, David. Doing qualitative research. London: Sage Publications, 2009.

${ }^{4}$ Trata-se de um conjunto de programas de computador voltados para auxiliar análises qualitativas. The Ethnographic, Atlas/Ti, NVivo, HyperResearch, MaxQda e WebQDA estão entre os mais utilizados nas áreas das Ciências Humanas e Sociais. Os pesquisadores britânicos Ray Lee e Nigel Fielding apresentaram o termo CAQDAS em 1989, em uma Conferência sobre Métodos de Pesquisa na Universidade de Surrey (UK), que desde 1994 abriga o CAQDAS Networking Project que fornece informações, treinamento e troca de experiências entre pesquisadores usuários desse tipo de programa computacional. Para maiores informações ver: http://www.surrey.ac.uk/ sociology/research/researchcentres/caqdas/about/index.htm

${ }^{5}$ Nos tempos, não tão distantes assim, em que os computadores pessoais e as rotinas informatizadas não eram tão comuns, muitos pesquisadores experientes não tinham familiaridade com essa nova linguagem e o principal referencial de gerenciamento informatizado de dados eram bancos de dados quantitativos. Dessa forma, muitos viam com reserva que o uso de programas de computador acabasse impondo uma lógica quantitativa no gerenciamento de dados qualitativos. Tal situação é improvável na medida em que os CAQDAS foram pensados levando em consideração justamente as especificidades e demandas das análises qualitativas (Baumgarten, Teixeira, Lima, 2007; Santos, 2001) .
} 
técnicas em si, como também suas implicações epistemológicas (Santos 2001; Silva, 1998). Afinal, as metodologias informacionais estariam imprimindo "um novo padrão de trabalho científico que revoluciona, porque altera, inverte e supera as modalidades vigentes de explicação sociológica" (Santos, 200, p.129).

\section{Sem fórmulas mágicas: os contras e os prós}

Nesse debate, os receios emergem da percepção de que os procedimentos informatizados poderiam provocar um engessamento do pesquisador, em alguma medida inibindo sua criatividade metodológica. Acerca dessa desconfiança dois pontos se destacam. O primeiro diz respeito à própria estrutura de um programa de computador, que é construído com base num conjunto de possibilidades para absorver, organizar e manusear informações. As demandas que orientaram os programadores na elaboração do produto não necessariamente correspondem às dos pesquisadores. Em alguns casos haveria de se adaptar as fontes à estrutura do software, adequando a um modelo de organização pré-estabelecido. Dessa forma, há uma percepção de que os pesquisadores deixariam de dedicar tempo e esforços em avaliações iniciais do material coletado para prontamente preparálo nos moldes exigido pelo programa escolhido (Silva, 1998; Lage, 2011).

A questão é relevante porque a forma como se organiza o material de pesquisa também produz efeitos sob a análise. Em versões antigas, os programas só aceitavam importação de arquivos em formato de texto (.txt) ou Rich Text Format (.rtf) que não permitiam formatações como parágrafos, notas, marcadores, entre outras. Exigia um esforço prévio de conversão bem cansativo e demorado, não sendo tão vantajoso em relação ao trabalho tradicional de organização e análise feito manualmente. Dessa maneira, a estrutura de um programa de computador pode significar uma camisa de força e levar a achados restritos, até mesmo 
induzidos ou ser uma lanterna a lançar luz para a exploração mais livre, flexível, dos conteúdos a serem analisados.

Dessa desconfiança decorre um segundo receio que é o de tornar o manuseio e, em consequência, a análise um conjunto de procedimentos padronizados e realizados mecanicamente. Isso pode vir a ser real se o programa de computador for entendido enquanto um fim e não um meio para a análise. Faz-se necessário a clareza de que se trata de uma ferramenta que se insere em um processo de trabalho que abriga vários níveis de tratamento das fontes. Um programa de computador pode facilitar e/ou agilizar algumas etapas, produzir menos papel, permitir organizar e relacionar grandes volumes e tipos variados de materiais, mas por si só não cria teorizações (Moreira, 2007; MacMillan, 2005; Azevedo, 1998; Baltar e Baltar, 2010; Lage, 2011).

Assim, o principal receio é pela adoção de uma novidade tecnológica sem a devida reflexão se ela realmente serve aos propósitos da pesquisa. De fato, seria pertinente a pergunta: seria um ferramental para ser usado por qualquer tipo de análise qualitativa? (MacMillan, 2005)

Os relatos de experiências de pesquisa que adotaram algum tipo de CAQDAS, ou até mesmo compararam programas diferentes, apresentam avaliações positivas sobre o armazenamento e as possibilidades de interação com materiais e documentos diversificados. Destaca-se a flexibilidade para operar todas as ações que se fariam manualmente, mas com a vantagem de poder articular um número maior de informações. Mas também se ressalta que é preciso ter clareza sobre o método de análise que se pretende realizar (análise do discurso; análise de conteúdo; análise interpretativa; geração de teoria...), assim como, proceder a uma boa avaliação de qual programa melhor servirá para realizar a estratégia de análise definida previamente. (MacMillan, 2005; Saillard, 2011; Macgilchrist e Van Hout, 2011; Silver e Patashick, 2011)

Vários autores discorrem que embora a estrutura da grande maioria desses programas seja semelhante, algumas rotinas ou procedimentos podem se mostrar mais fáceis, operacional ou 
| 78 |

Desafios metológicos da incorporação...

visualmente, em um determinado programa do que em outros (MacMillan, 2005; Moreira, 2007; Saillard, 2011; Macgilchrist e Van Hout, 2011; Silver e Patashick, 2011). Essa escolha não é considerada trivial, porque esbarra na falta de conhecimento em profundidade dessas ferramentas. Atualmente há disponível no mercado uma variabilidade não desprezível de programas e muito poucos usuários experientes. O que significa que invariavelmente, pesquisadores e equipes de pesquisa somente irão perceber os problemas reais durante o processo. Dessa maneira, investir no conhecimento prévio das vantagens e desvantagens de cada programa pode evitar frustrações e desgastes por trabalho desnecessário (Moreira, 2007; Saillard, 2011). E igualmente pode evitar o risco de adquirir uma bazuca para dar conta de um passarinho!

Os recursos desse ferramental, por outro lado, também alimentam expectativas muitas vezes equivocadas de encurtamento do tempo e dos esforços exigidos para uma análise qualitativa. Fazer com ajuda de um computador o trabalho que antes era feito manualmente com canetas coloridas, recortes e colagens, separando em pastas ou plásticos etiquetados segundo temas e categorias de análise, inegavelmente proporciona melhores condições para organizar, fragmentar e agregar em um único lugar - o seu computador pessoal - uma diversidade de tipos de materiais em análise. Como também facilita as idas e vindas entre suas partes e o todo. $\mathrm{O}$ que pode imprimir maior versatilidade e agilidade ao processo de análise. Contudo, o tempo dispendido e o trabalho exigido dependem da experiência de pesquisa, das habilidades e conhecimentos em informática dos pesquisadores envolvidos no processo (Baumgarten, Teixeira, Lima, 2007; Lage, 2011). O trabalho de se debruçar sobre o material continua sendo do mesmo tipo, apenas que passa a ser mediado por um computador. E que conforme o grau de familiaridade que se tem do programa que será utilizado pode exigir um investimento maior de tempo para aprender a direcionar os recursos do software para o tipo de tratamento que se quer dar aos dados (Lage, 2011; Baumgarten, Teixeira, Lima, 2007; Guizzo, Krziminski e Oliveira, 2003). Não 
raro desperta frustrações a constatação de que ter disponível um programa de computador para auxiliar a análise de dados não significa ter em mãos um oráculo que magicamente resolve todos os problemas de pesquisa (Baumgarten, Teixeira, Lima, 2007).

\section{O princípio da codificação: entre as partes e o todo}

Os programas criados para auxiliar análises qualitativas majoritariamente se baseiam no processo de codificação do material empírico. Criar códigos ou categorizações é parte integrante de grande parte dos modos de operacionalização de uma análise de fontes. Não há fórmula única para tal processo. Cada autor, movido por seus objetivos de pesquisa e arcabouços teóricos elabora um tipo de sistematização para criar e conceituar as categorias que utilizará ao longo do processo de refinamento da análise. Esse trabalho é muitas vezes iniciado no plano analítico esboçado ainda na metodologia do projeto e passará por inúmeras reelaborações ao longo da execução da pesquisa.

Os pressupostos teórico-metodológicos que orientam a lógica operacional dos CAQDAS é a grounded theory ${ }^{6}$, que implica em uma metodologia de identificação e definição de categorias analíticas mediante intensa e constante avaliação dos dados com a finalidade de gerar formulações teóricas substantivas. Trata-se de uma ação continuada de leitura, codificação, avaliação das informações trabalhadas e conclusões a partir dos dados categorizados, que por sua vez, dá início a um novo aprofundamento da leitura, codificação, avaliação e de resultados e assim por diante, em um ciclo que se repete até a consideração de que a análise adquiriu consistência e refinamento teórico (Moreira, 2007). Porém, quando esse processo é realizado sem uma sistemática reflexão crítica, o ato de codificar e recuperar informações pode se transformar em

${ }^{6}$ A Grounded theory se baseia nas elaborações dos sociólogos americanos Barney Glaser e Anselm Strauss que significa "descobrir uma teoria a partir dos fatos". (Moreira, 2007; Azevedo, 1998) 
| 80 |

Desafios metológicos da incorporação...

trabalho apenas descritivo, mecânico, volumoso ${ }^{7}$ e desprovido de uma interpretação substantiva dos dados. Novamente, é preciso lembrar que analisar dados vai muito além da sua codificação. (Moreira, 2007; MacMillan, 2005; Azevedo, 1998) Trata-se, como ressalta Santos (2001), de um processo em que se faz necessário exercitar a vigilância epistemológica: na atenção aos dados relevantes para a apreensão do objeto científico; na aplicação de um sistema para investigar e interpretar e na constante avaliação crítica desses procedimentos.

Inicialmente vários dos programas de tipo CAQDAS realizavam apenas procedimentos de codificação e busca, auxiliando na realização de fragmentações das fontes assim como igualmente facilitava a recuperação dos dados já categorizados e agregados. Como se tratam de softwares desenvolvidos a partir da interação contínua entre usuários, especialistas e programadores, muitas das limitações iniciais foram eliminadas e a cada nova versão outros recursos e demandas foram incorporados, imprimindo maior flexibilidade para o gerenciamento de dados qualitativos diversos (textos, vídeos, áudios e imagens). Grande parte da geração atual desses programas permite ampliar os horizontes da análise através de recursos que possibilitam estabelecer relações entre descritores ou fragmentações, gerar memorandos com anotações sobre conteúdos codificados, suas possíveis associações com outras fontes ou com elaborações teóricas (Fielding e Lee, 2002; Guizzo, Krziminski e Oliveira, 2003).

A fragmentação provocada pela codificação que retaliaria o material deslocando-o do seu contexto e prejudicando a análise do todo, integra grande parte das críticas aos programas, contudo, em vários deles a totalidade não se perde, na medida em que preservam o material armazenado em sua versão integral, podendo ser recuperado em qualquer fase do trabalho. Por

\footnotetext{
${ }^{7}$ Em algumas situações a codificação pode se tornar um trabalho obsessivo de categorizar tudo gerando uma quantidade excessiva e desnecessária de fragmentações e categorias sem sentido. (Moreira, 2007; MacMillian, 2005; Lage, 2011)
} 
exemplo, entrevistas realizadas no campo podem ser armazenadas enquanto áudio e texto transcrito. Em alguns programas quando o material é codificado, subarquivos são gerados com fragmentos que rementem as categorias. Ao trabalhar com esses subarquivos com fragmentos do material, se despertar alguma dúvida no pesquisador ou equipe de pesquisa que precise avaliar um contexto mais amplo, o documento original pode ser aberto simultaneamente para averiguações. A qualquer momento se pode fazer esse tipo de retomada do contexto mais geral. Como faríamos se estivéssemos analisando manualmente fitas cassetes e transcrições em papel.

Alguns autores (Lage, 2011; Guizzo, Krziminski e Oliveira, 2003; MacMillian, 2005; Teixeira e Becker, 2001) destacam essa questão, fornecendo exemplos bem concretos de estratégias e recursos via CAQDAS que podem dar uma clara ideia dos que se pode explorar de cada tipo de fonte na abordagem de temas diversos.

\section{Um horizonte de.... desafios}

Ao longo da última década impressiona o número de procedimentos e linguagens incorporados a cada nova versão dos programas mais populares (NVivo, MAXqda; Atlas/ti) ${ }^{8}$. Grande parte das atualizações acompanha as rápidas mudanças na linguagem da comunicação e das relações sociais mediadas pela informatização. Passa pela transformação visual, que se aproxima de programas com os quais já estamos bem familiarizados (em ambiente Windows) - facilitando o aprendizado e o domínio - até a importação de arquivos de texto, áudio, vídeo e outras mídias de qualquer extensão - que podem ser incorporadas internamente ou acessadas externamente (outros locais do computador ou na internet, por exemplo). Outro ponto que também merece destaque

\footnotetext{
${ }^{8}$ Em 2012, a QSR International que desenvolve o programa NVivo lançou sua versão número 10, disponível também em português pela primeira vez.
} 
são as possibilidades de combinação de estratégias metodológicas como aliar CAQDAS com Programas de Georeferenciamento (Fielding e Cisneros-Puebla, 2009) ou construção de análises qualiquanti. Para tais mudanças, a manutenção de uma intensa interlocução entre desenvolvedores, pesquisadores especialistas e usuários em geral tem sido considerada fundamental. A condução de fóruns especializados de discussões na internet e o incentivo a publicações com relatos de experiências são algumas das estratégias para a divulgação dessas ferramentas. Contudo, ainda se concentram nos centros de pesquisa europeus e norteamericanos.

Redes de sociabilidade virtuais, blogs e outras formas de comunicação via internet se unem às iconografias, vídeos, áudios, diários, registros manuscritos entre outros documentos e materiais comumente analisados nas pesquisas qualitativas. Tanto a incorporação dessas novas fontes documentais quanto programas de computador que auxiliem no armazenamento, organização e análise desses materiais tem desafiado os pesquisadores a alterar não apenas a maneira de trabalho, mas igualmente a desenvolver renovados olhares críticos sobre técnicas e métodos de análise, independente da adoção de uma perspectiva quantitativa ou qualitativa.

Estaríamos experimentando, segundo Santos (2001), uma inconclusa atualização do novo espírito científico, no qual a lógica interpretativa tem sido revolucionada pela multidimensionalidade permitida pelas ferramentas computacionais. Tanto no que diz respeito às fontes de pesquisa, ao tratamento analítico e apresentação dos resultados. Por essa razão, esse autor considera que ao contrário de inibir, as ferramentas computacionais podem aumentar a ousadia da imaginação sociológica no processo de construção do conhecimento.

Em suma, como bem destacam Teixeira e Becker (2001) e Baltar e Baltar (2010), programas de computador não podem ser vistos como oráculos de respostas prontas, seja para tratamento quantitativo ou qualitativo, são ferramentas que não prescindem do pesquisador. Afinal, as hipóteses, a criatividade, o olhar é 
sempre de quem realiza a pesquisa, que é quem igualmente definirá aquilo que deve ser examinado, agrupado, desmembrado, evidenciado e/ou omitido.

$\mathrm{O}$ desafio maior parece ser mais o da aprendizagem e domínio de novos modus operandis que propicie o desenvolvimento de abordagens que aproximem teoria e tratamento informacional. No entanto, deve advir de uma prática de ensino e pesquisa alimentada não apenas na pós-graduação, mas igualmente ao longo da formação das novas gerações de cientistas sociais, estimulando a renovação dos processos de aprendizagem e investigação.

\section{Bibliografia}

AZEVEDO, J. Programas de computadores para análises de dados qualitativos. In: ESTEVES, A; AZEVEDO, J. (eds.). In: Metodologias qualitativas para as Ciências Sociais, Porto: Universidade do Porto. Faculdade de Letras, 1998, pp. 149155.

BALTAR, R; BALTAR, C. S. A defasagem das ciências sociais no uso de recursos de informática para o ensino e a pesquisa no Brasil. In: La Educación - Revista Digital (OEA), v. 144, n. 2, 2010.

BAUMGARTEN, M; TEIXEIRA, A. N.; LIMA, G. Sociedade e conhecimento: novas tecnologias e desafios para a produção de conhecimento nas Ciências Sociais. In: Sociedade e Estado, v.22, n. 2, maio-ago, 2007; pp.401-433.

DWYER, T. As tecnologias de informação: morte ou vida para as ciências humanas? In: Sociologias, n. 12, jul/dez. 2004, pp. 328346.

FAZITO, D. Análises qualitativas na Demografia: um exercício de autocrítica dos fundamentos e práticas no campo demográfico. In: MIRANDA-RIBEIRO, Paula; SIMÃO, Andrea B. (orgs.) 
Desafios metológicos da incorporação...

Qualificando os números: estudos sobre saúde sexual e reprodutiva no Brasil. ABEP: UNFPA, Belo Horizonte, 2009, pp. 23-38.

FIELDING, N. G.; LEE, R, M. New patterns in the adoption and use of qualitative software. In: Field Methods, n. 14, 2002, pp.197216.

; CISNEROS-PUEBLA, C. A. CAQDAS-GIS Convergence. Toward a New Integrated Mixed Method Research Practice? In: Journal of Mixed Methods Research vol. 3, n. 4, oct, 2009, pp. 349-370.

GOLDENBERG, M. A Arte de Pesquisar: Como fazer pesquisa qualitativa em Ciências Sociais. Rio de Janeiro: Record, 2004. $8^{\text {a }}$ edição.

GUIZZO, B. S.; KRZIMINSKI, C. O.; OLIVEIRA, D. L. L. C. O software QSR NVivo 2.0 na análise qualitativa de dados: ferramentas para a pesquisa em ciências humanas e saúde. In: Revista Gaúcha de Enfermagem, vol. 24, n.1, 2003, pp.53-60.

LAGE, M. C. Utilização do software NVivo em pesquisa qualitativa: uma experiência em EaD. In: Educação Temática Digital, v.12, número especial, 2011, pp.198-226.

MACGILCHRIST. F; VAN HOUT, T. Ethnographic Discourse Analysis and Social Science. Forum Qualitative Research, v.12, n.1, 2011.

MACMILLAN, K. More than just coding? Evaluating CAQDAS in a discourse analysis of news texts. Forum Qualitative Research, v.6, n.3, 2005.

MARCONDES, G. S. O que um diário de campo tem a dizer sobre um questionário estruturado? Reflexões de uma experiência de estudo sobre grupos domésticos em Salvador, Bahia. In: CONGRESO DE ALAP, 4, 2010, Habana. Anales... Rio de Janeiro: Asociación Latinoamericana de Población, 2010. 
MOREIRA, D. A. O uso de programas de computador na análise qualitativa: oportunidades, vantagens e desvantagens. Revista de Negócios, v.12, n.2, abr-jun, 2007, pp. 56-58.

SAILLARD, E. K. Systematic Versus Interpretive Analysis with two CAQDAS Packages: NVivo and MAXQDA. Forum Qualitative Research, v.12, n.1, 2011.

SANTOS, J.V.T.As possibilidades das metodologias informacionais nas práticas sociológicas: por um novo padrão de trabalho para os sociólogos do Século XXI. In: Sociologias, n.5, jan-jun, 2001, pp. 116-146.

SILVA, E. A. Bancos de dados e pesquisa qualitativa em História: reflexões acerca de uma experiência. Revista de História Regional, vol. 3, n.2, 1998, pp. 167-176.

SILVER, C. PATASHNICK, J. Finding Fidelity: Advancing Audiovisual Analysis Using Software. Forum Qualitative Research, v.12, n.1, 2011.

SOUZA, F. N.; COSTA, A. P.; MOREIRA, A. Questionamento no Processo de Análise de Dados Qualitativos com apoio do software WebQDA. In: EduSer. Revista de Educação, Inovação em Educação com TIC. v. 3, n. 1, 2011, pp. 19-30.

TEIXEIRA, A. N.; BECKER, F. Novas possibilidades da pesquisa qualitativa via sistemas CAQDAS. In: Sociologias, n.5, jan-jun, 2001, pp. 94-113. 